\title{
Cost-outcome description of clinical pharmacist interventions in a university teaching hospital
}

\author{
James Gallagher ${ }^{1,4^{*}}$, Stephen Byrne ${ }^{1}$, Noel Woods ${ }^{2}$, Deirdre Lynch ${ }^{3}$ and Suzanne McCarthy ${ }^{1}$
}

\begin{abstract}
Background: Pharmacist interventions are one of the pivotal parts of a clinical pharmacy service within a hospital. This study estimates the cost avoidance generated by pharmacist interventions due to the prevention of adverse drug events (ADE). The types of interventions identified are also analysed.

Methods: Interventions recorded by a team of hospital pharmacists over a one year time period were included in the study. Interventions were assigned a rating score, determined by the probability that an ADE would have occurred in the absence of an intervention. These scores were then used to calculate cost avoidance. Net cost benefit and cost benefit ratio were the primary outcomes. Categories of interventions were also analysed.

Results: A total cost avoidance of $€ 708,221$ was generated. Input costs were calculated at $€ 81,942$. This resulted in a net cost benefit of $€ 626,279$ and a cost benefit ratio of 8.64: 1. The most common type of intervention was the identification of medication omissions, followed by dosage adjustments and requests to review therapies.

Conclusion: This study provides further evidence that pharmacist interventions provide substantial cost avoidance to the healthcare payer. There is a serious issue of patient's regular medication being omitted on transfer to an inpatient setting in Irish hospitals.
\end{abstract}

Keywords: Hospital pharmacy, Adverse drug events, Health care economics, Ireland, Clinical pharmacy services, Cost avoidance

\section{Background}

The traditional role of a pharmacist predominantly involved the dispensing of medications in both hospital and community settings; consequently the pharmacist was quite detached from other healthcare professionals. The profession has since evolved to become recognised as an essential part of the healthcare team [1]. While still ensuring that medicines are sourced and dispensed to the highest possible standards, pharmacists have diversified into alternative areas of care in hospital practice [2]. Interventions are integral components of the new enhanced role which pharmacists offer in a clinical setting [3-8].

A pharmacist intervention is defined as any action taken by a pharmacist that aims to change patient management or therapy [9]. A pharmacist's expertise in pharmacology, pharmacotherapy and pharmaceutics ensures they have the

\footnotetext{
* Correspondence: j.e.gallagher@umail.ucc.ie

'Clinical Pharmacy Research Group, School of Pharmacy, University College Cork, Cork, Ireland

${ }^{4}$ HRB Clinical Research Facility at UCC, Mercy University Hospital, Cork, Ireland Full list of author information is available at the end of the article
}

requisite capabilities to offer suggestions to other healthcare staff on possible alterations to a patient's therapy $[10,11]$. This helps to ensure optimal patient outcomes, which has the potential to have an add-on economic benefit to the healthcare institution.

A myriad of studies have described the high rate of potential inappropriate prescribing and potential adverse drug events (ADE) in multiple healthcare systems [12-16]. An ADE is defined by the International Conference for Harmonisation as "any untoward medical occurrence in a patient administered a medicinal product which does not necessarily have to have a causal relationship with treatment". These issues cause repercussions in the form of increased resource utilisation [17]. Evidence of the clinical benefit and reduction in ADEs associated with enhanced roles for pharmacists in a hospital setting, are documented in the literature $[2,5,18,19]$.

Healthcare systems worldwide are coming under increasing pressure due to a combination of aging populations and the proliferation of new expensive technologies 
[20]. All healthcare services need to show that they provide value for money for the investment made in their provision [21]. Despite early scepticism, health economic evaluations are required for the establishment and continued provision of services and technologies [20].

The provision of a clinical pharmacy service in a hospital setting is an investment which utilises costs which could be used elsewhere in the health system. Economic evaluations of clinical pharmacy services will help policy makers make informed decisions on whether they are a worthwhile investment. Studies in other jurisdictions have indicated that they are cost-effective, however these findings are not generalisable [22,23].

This paper will analyse the interventions made by a team of pharmacists in a university teaching hospital and evaluate the cost avoidance achieved through the prevention of an ADE. For the purposes of this study, cost avoidance refers to an intervention that reduces or eliminates additional expenditure that otherwise may have been incurred in the absence of the intervention [24]. It is a different measure to cost saving interventions, which refer to reductions in current spending due to changes in the expenditure on a patients treatment [25]. Cost avoidance interventions contain and control costs and over a longer period of time they can result in cost savings.

The study was based in an Irish university teaching hospital/tertiary referral centre setting over a period of one year. Similar studies have been performed, examining shorter time periods or focusing on specific interventions [3-7,25]. However, information which helps to evaluate the economic impact of pharmacist interventions over a longer period of time and covering an entire hospital domain is lacking. The authors have been unable to find a study where cost avoidance generated by a full department of clinical pharmacists in a full calendar year has been calculated.

\section{Methods \\ Setting}

This was a retrospective study based on a 1 year time period from 01/01/2012 to 31/12/2012 inclusive. Cork University Hospital (CUH) and the Cork University Maternity Hospital is a combined 850-bed hospital site. The hospital serves a population of over 620,000 . In addition, it is a tertiary referral centre for over 1 million people, in the southern region of the Republic of Ireland. Approximately 32,000 inpatient admissions were recorded for 2012. All patients who were in receipt of a pharmacist intervention on their drug therapy were included in this study. There were no additional exclusion criteria. The Pharmacy Department consists of 15.8 whole time equivalent (WTE) pharmacists. As two WTEs are employed in managerial and administrative capacities, 13.8 WTE are available to document interventions as part of the daily pharmaceutical service. Interventions performed in all areas of the hospital, including the maternity unit, were included in this study.

\section{Intervention analysis}

Clinical pharmacist interventions are provided by many basic and senior grade pharmacists at CUH. Clinical pharmacist interventions are carried out at patient admission, during pharmacist led patient chart review or at the request of another healthcare professional. As previously discussed, the primary goal of a clinical pharmacist intervention is to ameliorate patient therapy.

Interventions made by pharmacists were recorded on a duplicate paper form. One form is kept by the pharmacist and one is passed on to the attending physician who has the final decision on whether to accept or reject the intervention.

In addition to producing a paper record of the intervention, the pharmacist retrospectively enters the proposed intervention into the 'eClinical Pharmacy Suite'. The 'eClinical Pharmacy Suite' is a browser-based application, aimed at supporting clinical pharmacists to record, grade and report medication related interventions or errors. Interventions were assigned by the clinical pharmacist to the most appropriate category in the application. Due to time constraints, not all recorded intervention had been entered on the computerised database. The primary researcher inputted any outstanding interventions and verified data which had been previously entered by hospital pharmacists. Intervention categories were developed based on the recommendations of an advisory group of clinical pharmacists from Ireland.

\section{Cost analysis}

The cost of providing this service was calculated based on the average time it took to carry out an intervention and the hourly cost of employing a hospital pharmacist. The average time of an intervention was based on a previously published study, which showed that the majority of pharmacist interventions in a university hospital setting took between 15 - 30 minutes to complete [7]. The hourly rate of employing a pharmacist at the mid-point of the salary scale was calculated based on an annual salary of $€ 49,425$ (Point 6 of 2010 salary scale) [26]. This underwent upward revision to account for employer related costs and hospital overheads based on guidance for conducting an economic analysis within the Irish healthcare system [27-29]. Base case scenario was calculated using the hourly cost of employing a basic level pharmacist at the mid-point of the salary scale and an average intervention time of 22.5 minutes.

Cost avoidance was calculated based on the probability that an ADE would have occurred in the absence of the proposed pharmacist intervention [18]. Interventions were 
analysed by the primary author and a score was assigned based on the probability of a patient experiencing harm directly or indirectly from their prescribed/administered medicines, and also the potential of omission of regular medication, sub-therapeutic dosing or an ill-advised choice of therapy. Determination of the probability that a patient would experience harm in the absence of an action by a pharmacist was based on the methodology described by Nesbit et al. [25] (Table 1). The cost avoidance for each individual intervention was determined and total cost avoidance was accordingly calculated through summation of the individual interventions. A random sample of the interventions $(n=100)$ were reviewed by two academic pharmacists with hospital pharmacy experience and inter-rater reliability was calculated for the sample.

The authors were unable to find an estimated cost of an $\mathrm{ADE}$, calculated based on data from the Irish healthcare system. The additional cost of treating an inpatient that experienced an ADE was taken from a recent study which utilised a micro-costing approach based on data from German hospitals [30]. This study also included a range of previously published ADE estimates (€934 - 5783). The majority of previously published studies which calculate cost avoidance used a cost of ADE determined by Bates et al. [5,7,24,25]. Rottenkolber ADE valuation was deemed to be more appropriate for this study as it was published in 2012 while Bates study was published in 1997 using US data [31]. This removed the need to account for currency differentials and inflation. Purchasing power parities between Germany and Ireland were used to further minimise differentials between the two countries. Cost of an ADE used in base case scenario was $€ 1057$.

A micro-costing approach, assigns a valuation to each individual unit of resource consumed and is considered the most robust costing method [32]. Diagnosis related group (DRG) costs for toxic side effects of drugs based on Irish hospital data were available but were not chosen as the cost of an ADE. The DRG estimate exclusively measured toxic side effects of drugs [33], furthermore DRG costs are generally less accurate in comparison to micro-costing estimates [32]. DRG costs for toxic side effects for drugs ( $€ 887)$ were included in sensitivity analysis calculations.

Following estimation of the cost of carrying out the pharmacist interventions and the resulting cost avoidance, net cost benefit and cost benefit ratio for providing the service were calculated. Analysis was calculated from the perspective of the healthcare institution. Discounting was excluded as events were all considered to have taken place in a 1 year time period.

\section{Sensitivity analysis}

One way deterministic sensitivity analysis was performed. Published ranges and confidence intervals where available determined the extent of the parameters. Sensitivity analysis was also performed using alternative published costs of an ADE [31], Irish DRG data and with various intervention acceptance rates.

\section{Data analysis}

Reports generated from the eClinical Pharmacy Suite database were in Microsoft Excel ${ }^{\mathrm{TM}}$ format. Summary statistics were calculated through Microsoft Excel 2010 (Microsoft Corp., Redmond, Washington). All other advanced analysis was conducted through IBM SPSS Statistics Version 18.

\section{Ethical approval}

Approval was obtained from the Clinical Research Ethics Committee of the Cork Teaching Hospitals, University College Cork, Ireland.

\section{Results}

A total of 4,257 interventions were documented on 2,147 individual patients (Table 2). The majority of the interventions were judged to have prevented potential

Table 1 Nesbit method for calculating indirect cost benefit [25]

\begin{tabular}{|c|c|c|}
\hline \multirow{2}{*}{$\begin{array}{l}\text { Equation 1: Cost avoidance } \\
\text { for individual intervention } \\
\text { Probability of an ADE occurring }\end{array}$} & \multicolumn{2}{|c|}{$\begin{array}{l}\text { Probability of an } A D E \text { occurring } X \text { cost of an } A D E[30] \\
\text { OR ( } 0 \text { or } 0.01 \text { or } 0.1 \text { or } 0.4 \text { or } 0.6) X € 1057\end{array}$} \\
\hline & Probability score & Example \\
\hline No harm expected & 0.00 & $\begin{array}{l}\text { Pharmacist suggests changing patient from esomeprazole } \\
\text { to omeprazole exclusively for economic reasons. }\end{array}$ \\
\hline Very low & 0.01 & $\begin{array}{l}\text { Patient regularly takes a bisphosphonate, but medication } \\
\text { omitted from hospital kardex }\end{array}$ \\
\hline Low & 0.10 & $\begin{array}{l}\text { Patient takes an antibiotic twice daily, when recommended } \\
\text { dose would be three times daily. }\end{array}$ \\
\hline Medium & 0.40 & $\begin{array}{l}\text { Metformin dose not reduced despite patient demonstrating } \\
\text { renal impairment. }\end{array}$ \\
\hline High & 0.60 & $\begin{array}{l}\text { Patient prescribed amiodarone while currently taking digoxin } \\
\text { without any reduction in digoxin dose. }\end{array}$ \\
\hline
\end{tabular}


Table 2 Intervention analysis

\begin{tabular}{ll}
\hline Total number of patients who received intervention & $\begin{array}{l}\text { Number of } \\
\text { interventions }\end{array}$ \\
Total number of interventions & 2147 patients \\
Mean number of interventions per patient (St. dev.) & 4257 \\
Median number of interventions per patient (95\% Cl) & $1.98(1.60)$ \\
Range of interventions per patient & $1-18$ \\
Interventions accepted by physicians (\%) & $1275(29.92)$ \\
Interventions rejected by physicians (\%) & $61(1.43)$ \\
Interventions with unknown acceptance outcome (\%) & $2921(68.81)$ \\
\hline
\end{tabular}

ADEs $(n=3,417)$. The remaining interventions had no discernible impact on therapy or patient outcomes, based on the judgement of the primary author. Additional interventions required entry under multiple categories on the database, but were only evaluated once for potential prevention of an ADE.

Recorded acceptance rate by physicians was $29.92 \%$ $(\mathrm{n}=1275)$. Only $1.43 \%(\mathrm{n}=61)$ interventions were recorded as being rejected by a physician. However, the rate of interventions with an unknown acceptance outcome was high, 68.81\% $(\mathrm{n}=2921)$.

Substantial cost avoidance of $€ 710,000$ was generated over a 1 year period from the perspective of the health care provider. Mean cost avoidance of $€ 166$ per intervention was generated. The cost of providing these interventions was $€ 82,000$. Substantial net cost benefits of $€ 626,279$ and a cost benefit ratio of 8.64 were generated based on this evaluation of pharmacist interventions (Table 3).

The number of interventions that potentially avoided ADEs were as follows: 119 (2.8\% of all interventions) of the interventions were associated with a probability score of 0.6 (high likelihood of preventing an adverse event), 1101 interventions (25.86\%) were associated with a probability score of 0.4 (medium), 1514 interventions (35.56\%)

Table 3 Cost analysis of pharmacist interventions

\begin{tabular}{|c|c|c|}
\hline & & $\begin{array}{r}\text { Base case } \\
\text { (Range) }\end{array}$ \\
\hline 1. & Cost avoidance & $\begin{array}{r}\boldsymbol{\epsilon 7 0 8 2 2 1} \\
(625808-3874783)\end{array}$ \\
\hline 2. & $\begin{array}{l}\text { Cost of Service } \\
\text { - Pharmacist Wages }\end{array}$ & $\begin{array}{r}€ \mathbf{8 1 9 4 2} \\
(22402-110389)\end{array}$ \\
\hline${ }^{a} 3=(1-2)$ & Net Cost Benefit & $\begin{array}{r}\boldsymbol{\epsilon 6 2 6 2 7 9} \\
(567173-3764394)\end{array}$ \\
\hline${ }^{b} 4=(1 / 2)$ & Cost Benefit Ratio $\frac{\text { Net Cost Benefit }}{\text { Cost of Senvice }}$ & 8.64 \\
\hline
\end{tabular}

were associated with a probability score of 0.1 (low), 683 interventions $(16.04 \%)$ were associated with a probability score of 0.01 (very low) and 840 interventions $(19.73 \%)$ were associated with a probability score of 0 (no harm expected).

The most prevalent type of intervention was the identification of omissions of patient's regular pre-admission medication, followed by requests to change the dose of medications and requests for the physician to consider whether it was appropriate to continue with a medication (Table 4). The most common categories of medications to require interventions were proton pump inhibitors $(n=259)$, statins $(n=208)$, beta-blockers $(n=165)$, corticosteroids $(n=161)$ and penicillins $(n=157)$.

Inter rater reliability indicated an acceptable level of agreement, based on a random sample of 100 interventions. Average agreement between 3 raters was 0.744 . Individual pairwise agreement was over the significant level of 0.7 for all 3 comparisons: Primary Rater (PR) Academic Pharmacist (AP) $1=0.763$, PR - AP $2=0.761$, AP 1 - AP $2=0.709$.

In all scenarios examined, the cost-benefit ratio remained positive (Table 5). All known variables underwent a oneway sensitivity analysis based on known ranges or using variations used in previously published sensitivity analysis on the topic. Nesbit et al. conducted a sensitivity analysis where the ADE probability underwent an each way variation of $50 \%$, an identical variation was undertaken in this paper [25]. The greatest variance in cost-benefit ratio was displayed in the cost assigned to an ADE.

Two additional ADE cost estimates were investigated during the course of sensitivity analysis (Table 6). The estimated cost of an ADE calculated by Bates and adjusted due to change in setting and year resulted in a considerable increase in the cost benefit ratio.

Table 4 Intervention categorisation

\begin{tabular}{ll}
\hline Category of intervention & N (\%) \\
\hline Drug & $2759(64.81 \%)$ \\
- Drug, Omissions & $1820(65.93 \%$ of Drug Category) \\
- Drug, Review Therapy & $421(15.24 \%$ of Drug Category) \\
- Drug, Interaction & $124(4.56 \%$ of Drug Category) \\
- Drug, Other & $394(14.27 \%$ of Drug Category) \\
Doses & $920(21.61 \%)$ \\
Frequencies & $354(8.32 \%)$ \\
Routes & $125(2.94 \%)$ \\
Duration & $47(1.10 \%)$ \\
Other & $27(0.63 \%)$ \\
Date/Time & $20(0.47 \%)$ \\
Rates & $5(0.12 \%)$ \\
\hline
\end{tabular}


Table 5 Sensitivity analysis for cost-benefit ratios

\begin{tabular}{|c|c|c|}
\hline Variable & Lower limit (Cost Benefit Ratio) & Upper limit (Cost Benefit Ratio) \\
\hline Time & 30 minutes per intervention 6.48 & 15 minutes per intervention 12.96 \\
\hline ADE Probability & $-50 \%$ Probability score 4.32 & $\mathbf{+ 5 0 \%}$ Probability score 12.96 \\
\hline Salary & Highest point on senior pharmacist scale 6.42 & Lowest point on basic pharmacist scale 12.07 \\
\hline $\operatorname{ADE} \operatorname{Cost}^{\mathrm{A}}$ & Lowest point on range 7.63 & Highest point on range 47.28 \\
\hline Intervention acceptance & 50\% Acceptance 4.32 & Known Acceptance (29.92\%) 2.59 \\
\hline
\end{tabular}

${ }_{\mathrm{A}}^{\mathrm{A} D E}$ range taken from a review of selected international studies regarding the economic consequences of ADEs which reported additional mean costs in the range of $€ 934$ to $€ 5783$ per case [34].

\section{Discussion}

Substantial cost avoidance was demonstrated in this study. Cost benefit ratio and net cost benefit remained positive under all conditions examined. To the authors' knowledge, this study is the first that has attempted to estimate the cost avoidance achieved in a total hospital environment over such an extended period of time. Direct comparison of savings generated in this study with previously published studies is difficult. The generalizability of pharmacoeconomic analysis is uncertain [35]. Calculation of cost avoidance will have inter study variations in the cost assigned to an $\mathrm{ADE}$, methodologies, healthcare settings, duration of study and number of pharmacists employed.

The original study which implemented the cost avoidance method used in this paper generated a net benefit of $\$ 392,660$ [25]. This was over a similar 12 month period but only included 3 WTE pharmacists operating on three specific hospital wards. Study location was in a US hospital. These pharmacists had undergone specialist training and were to a large extent exclusively performing interventions during their participation in the study. Cost benefit ratio of 3.1:1 was considerably smaller in the Nesbit study. Cost-benefit ratio was influenced significantly by the method used to calculate the cost of the service. The complete pharmacist salary was used to calculate the cost of providing the intervention rather than apportioning part of the salary based on the time pharmacist spent enacting the interventions.

A study by Olson et al. conducted in a 360 bed US hospital has the greatest methodological agreement with this paper [7]. This was conducted over a 3 month period and estimated the cost benefit ratio to be 1.2. Only 5 pharmacists provided interventions in this study. Although, substantial savings $(\$ 84,631)$ was generated from a small number of interventions, the cost-benefit ratio was lower than expected. As with the Nesbit study, cost-benefit ratio was reduced as full pharmacist salary was used to

Table 6 Sensitivity analysis using alternative ADE estimates

\begin{tabular}{lr}
\hline Alternative ADE estimate & Cost benefit ratio \\
\hline DRG Toxic Side Effects [33] & 7.25 \\
Bates ADE Costing [31] & 49.49 \\
\hline
\end{tabular}

calculate the cost of providing the service. The results in this study provides evidence that the positive cost benefit ratio of clinical pharmacists' interventions is maintained over a longer period of time, with additional pharmacists and in a wider hospital setting.

Cost avoidances are impacted significantly if they are focused in specific departments. Cost avoidance per intervention generated in our study was decidedly lower in comparison to a study conducted in an intensive care unit. The addition of a single critical care pharmacist to a 16 bed intensive care unit produced an average cost avoidance of \$1596.27 - \$1615.67 per intervention [5]. Input costs were omitted in this study so further comparison was not feasible.

While the cost-benefit ratio in this study was positive, it needs to be reiterated that this ratio was based on estimates of time and avoidance of cost rather than hard economic data. Therefore, the ratio could potentially be an overestimate. Furthermore, an evaluation of a clinical pharmacy service is strengthened when it also includes an assessment of the clinical and humanistic outcomes involved [36].

This high degree of omissions of patient's regular pre-admission medications (43\% of all interventions) highlights the need for a dedicated system of medicines reconciliation at a tertiary healthcare level in Ireland. A pilot study of pharmacist-led medication reconciliation in two university teaching hospitals, elsewhere in Ireland, also identified omission of a pre-admission medication as the most common discrepancy [37]. These findings are replicated in other healthcare settings [38]. Medication omissions can have potentially serious consequences for patients depending on the nature of the drug omitted [39].

An argument could be presented not to include medication omissions as an ADE, based on a strict interpretation of the definition, as the patient did not receive the drug [40]. However, the decision was taken to include them in this study. Similar studies in the past have included the identification of medication omissions as a potential ADE $[7,41]$. The probability of a patient incurring harm is increased if they do not receive their regular medication, resulting in a related increase in hospital resource utilisation [42]. 
Medium and low scores were the most frequent probabilities assigned to the interventions. This reflects findings obtained in the majority of previous studies which implemented the same method of calculating cost avoidance $[5,25,41]$. The frequency of these scores were influenced by the number of medication omissions (39\% of omissions were assigned a medium probability score and $37 \%$ assigned a low probability score.) Interventions designated with a high probability of preventing an $A D E$ were largely composed of omissions of essential medications (e.g. anti-epileptic drugs) or known serious drug interactions.

The most common medication classifications requiring intervention were unsurprising. Proton pump inhibitors (PPI) were the medication classification requiring the most frequent intervention. Interventions on PPIs were largely suggestions to change to lower cost equivalents, highlighting therapeutic duplication, excess of therapy or suggestion for switching from intravenous to oral administration. The majority of these interventions resulted in cost savings to the healthcare provider. Inappropriate prescribing of PPIs is a significant issue in the Irish healthcare system and a significant drain on resources [43,44]. This demonstrates the pivotal role can play as cost containers in the healthcare system.

Another method of increasing the number of interventions would be the provision of further training for clinical pharmacists which would enable them to become specialists in various areas of patient care. This practice is common in other countries and has been shown to provide substantial monetary savings from interventions enacted by specialist pharmacists [5]. Specialised anti-microbial pharmacists have been shown to be of value and are now established in many hospitals in Ireland [45,46].

A significant level of agreement was found between three of the listed authors. Assignment of probabilities is subjective; therefore a high level of agreement is unlikely. On review of samples, almost all were within 1 score of each other, further indicating that probabilities were assigned by the primary author in a manner consistent with fellow professionals. Although, inter rater reliability was not examined for all interventions, the clinical background of the primary rater and the significant agreement level demonstrated in the sample indicate the ADE probabilities were assigned in an appropriate and consistent manner.

There was a high rate of interventions where the outcome was unknown at time of analysis. However, review of interventions where status was determined indicated that only a small minority of pharmacist interventions were rejected. This indicates that other healthcare professionals are receptive to pharmacist interventions on patient medication. The high level of unknown outcomes was most likely due to time constraints on the pharmacist. Following acceptance or rejection, system requires manual updating. It is understandable that an administrative task such as this may be neglected.

The importance of an accurate estimation of ADE cost was emphasized from the dramatic increase in cost benefit ratio if Bates et al. estimate of ADE was used. As previously stated, there is an absence of data on ADE costs in Ireland. The validity of estimated cost avoidance would be improved through application of local data on excess costs associated with ADE. Until this issue has been addressed, imperfect data is the only viable option. Sensitivity analysis undertaken in this study was deterministic in nature; performing probability sensitivity analysis would have been a more accurate robust method to determine whether pharmacist interventions would have maintained a positive cost benefit ratio.

The primary limitation was the limited availability of additional patient information (medical record, medical history, outcome of intervention etc.) when assigning adverse drug event probabilities. As this study contained a large number of patient interventions, it was not feasible to retrospectively retrieve this information from medical notes.

Another major limitation was the exclusion of some potential input costs. In order to conduct an intervention, problems must be located which takes up pharmacist time and therefore has an associated cost. Screening of a patient's medication may not be exclusively for the purpose of discovering interventions but it is one possible outcome from it. Additionally, other healthcare professionals are required to spend time reviewing suggested pharmacist interventions. This was also excluded from analysis.

Utilisation of a scoring system which also accounts for the severity of the potential ADE would significantly enhance this study. Such scoring systems exist but they do not assign a cost with the scoring outcome generated. There is no ideal system for assigning probabilities to adverse events but the widespread adaptation of one system would add to the ability to compare studies across jurisdictions. The classification of interventions is subjective. Generation of local guidelines on the classification of interventions would help reduce this variation.

While the interventions included in the study represented the majority of work conducted by clinical pharmacists, it is possible that some interventions were not inputted on to the eClinical System. Therefore, the current data may under-represent the cost avoidance produced.

The interventions were assigned scores by an individual rater. While sample of interventions examined for inter-rater reliability indicated that the primary author was assigning probability scores in a manner consistent with other pharmacists, review of complete dataset by additional pharmacists and other medical professionals would have enhanced the study. 


\section{Conclusion}

Previous reviews have indicated that pharmacist interventions generate significant cost avoidance when measured under certain criteria and conditions [18]. This study has confirmed previous opinions and supplemented the body of evidence that the provision of clinical pharmacy services in an entire hospital provides value for money to the healthcare payer. An excessive amount of omissions of regular medications has been highlighted by this study. The estimation of cost avoidance would be improved by the development of a method which incorporated the potential severity of an ADE into the evaluation and an evaluation of excess costs associated with ADEs in an Irish healthcare setting.

\section{Competing interests}

The authors declare that they have no competing interests.

\section{Authors' contributions}

$J G$ performed analysis on data and was the primary author of the manuscript. SB devised the concept for the manuscript, performed analysis on sample data and revised drafts of the manuscript. NW provided advice on appropriate methods for calculating costs and revised drafts of the manuscript. DL revised drafts of the manuscript. SMcC participated in data collection, performed analysis on sample data and revised drafts of the manuscript. All authors read and approved the final manuscript.

\section{Acknowledgements}

We would like to thank the staff at the Pharmacy Department, Cork University Hospital for their assistance. We would also like to thank Caroline Reidy of Pfizer Healthcare Ireland, for provision of the eClinical Pharmacy Suite software to the Pharmacy Department at Cork University Hospital. Funding for this research was kindly provided by the HRB-Clinical Research Facility (UCC).

\section{Author details}

${ }^{1}$ Clinical Pharmacy Research Group, School of Pharmacy, University College Cork, Cork, Ireland. ${ }^{2}$ Centre for Policy Studies, University College Cork, Cork, Ireland. ${ }^{3}$ Pharmacy Department, Cork University Hospital, Cork, Ireland. ${ }^{4} \mathrm{HRB}$ Clinical Research Facility at UCC, Mercy University Hospital, Cork, Ireland.

\section{Received: 20 November 2013 Accepted: 25 March 2014}

Published: 17 April 2014

\section{References}

1. Halvorsen $\mathrm{KH}$, Stensland P, Granas AG: A qualitative study of physicians' and nurses' experiences of multidisciplinary collaboration with pharmacists participating at case conferences. Int J Pharm Pract 2011, 19(5):350-357.

2. Kaboli PJ, Hoth AB, McClimon BJ, Schnipper JL: Clinical pharmacists and inpatient medical care: a systematic review. Arch Intern Med 2006, 166(9):955-964.

3. Khalili H, Karimzadeh I, Mirzabeigi P, Dashti-Khavidaki S: Evaluation of clinical pharmacist's interventions in an infectious diseases ward and impact on patient's direct medication cost. Eur J Intern Med 2013, 24(3):227-233.

4. Klopotowska JE, Kuiper R, van Kan HJ, de Pont AC, Dijkgraaf MG, Lie-A-Huen L, Vroom MB, Smorenburg SM: On-ward participation of a hospital pharmacist in a Dutch intensive care unit reduces prescribing errors and related patient harm: an intervention study. Crit Care 2010, 14(5):R174.

5. Kopp BJ, Mrsan M, Erstad BL, Duby JJ: Cost implications of and potential adverse events prevented by interventions of a critical care pharmacist. Am J Health Syst Pharm 2007, 64(23):2483-2487.

6. Lada P, Delgado G Jr: Documentation of pharmacists' interventions in an emergency department and associated cost avoidance. Am J Health Syst Pharm 2007, 64(1):63-68.

7. Olson LM, Desai S, Soto ML, Namazifard S, Quelland AK, Erstad BL: Evaluation of pharmacists' interventions at a university teaching hospital. Can J Hosp Pharm 2005, 58(1):20-25.
8. Shen J, Sun Q, Zhou X, Wei Y, Qi Y, Zhu J, Yan T: Pharmacist interventions on antibiotic use in inpatients with respiratory tract infections in a Chinese hospital. Int J Clin Pharm 2011, 33(6):929-933.

9. Alderman CP, Farmer C: A brief analysis of clinical pharmacy interventions undertaken in an Australian teaching hospital. J Qual Clin Pract 2001, 21(4):99-103.

10. Marriott JL, Nation RL, Roller L, Costelloe M, Galbraith K, Stewart P, Charman WN: Pharmacy education in the context of Australian practice. Am J Pharm Educ 2008, 72(6):131.

11. Sosabowski MH, Gard PR: Pharmacy education in the United Kingdom. Am J Pharm Educ 2008, 72(6):130.

12. Bradley MC, Fahey T, Cahir C, Bennett K, O'Reilly D, Parsons C, Hughes CM: Potentially inappropriate prescribing and cost outcomes for older people: a cross-sectional study using the Northern Ireland Enhanced Prescribing Database. Eur J Clin Pharmacol 2012, 68(10):1425-1433.

13. Budnitz DS, Lovegrove MC, Shehab N, Richards CL: Emergency hospitalizations for adverse drug events in older Americans. N Engl J Med 2011, 365(21):2002-2012

14. Dequito AB, Mol PG, van Doormaal JE, Zaal RJ, van den Bemt PM, Haaijer-Ruskamp FM, Kosterink JG: Preventable and non-preventable adverse drug events in hospitalized patients: a prospective chart review in the Netherlands. Drug Saf 2011, 34(11):1089-1100.

15. O'Sullivan DP, O'Mahony D, Parsons C, Hughes C, Murphy K, Patterson S, Byrne $S$ : A prevalence study of potentially inappropriate prescribing in Irish long-term care residents. Drugs \& aging 2013, 30(1):39-49.

16. Ryan C, O'Mahony D, Kennedy J, Weedle P, Byrne S: Potentially inappropriate prescribing in an Irish elderly population in primary care. Br J Clin Pharmacol 2009, 68(6):936-947.

17. Classen DC, Pestotnik SL, Evans RS, Lloyd JF, Burke JP: Adverse drug events in hospitalized patients. Excess length of stay, extra costs, and attributable mortality. JAMA 1997, 277(4):301-306.

18. De Rijdt T, Willems $L$, Simoens S: Economic effects of clinical pharmacy interventions: a literature review. Am J Health Syst Pharm 2008, 65(12):1161-1172.

19. Dooley MJ, Allen KM, Doecke CJ, Galbraith KJ, Taylor GR, Bright J, Carey DL: A prospective multicentre study of pharmacist initiated changes to drug therapy and patient management in acute care government funded hospitals. Br J Clin Pharmacol 2004, 57(4):513-521.

20. Hughes DA: From NCE to NICE: the role of pharmacoeconomics. Br J Clin Pharmacol 2010, 70(3):317-319.

21. Hughes DA: Pharmacoeconomics. Br J Clin Pharmacol 2012, 73(6):968-972

22. Bond CA, Raehl CL: Clinical pharmacy services, pharmacy staffing, and hospital mortality rates. Pharmacotherapy 2007, 27(4):481-493.

23. MacLaren R, Bond CA, Martin SJ, Fike D: Clinical and economic outcomes of involving pharmacists in the direct care of critically ill patients with infections. Crit Care Med 2008, 36(12):3184-3189.

24. Campbell AR, Nelson LA, Elliott E, Hieber R, Sommi RW: Analysis of cost avoidance from pharmacy students' clinical interventions at a psychiatric hospital. Am J Pharm Educ 2011, 75(1):8.

25. Nesbit TW, Shermock KM, Bobek MB, Capozzi DL, Flores PA, Leonard MC, Long JK, Militello MA, White DA, Barone LD, Goldman MP, Kvancz DA: Implementation and pharmacoeconomic analysis of a clinical staff pharmacist practice model. Am J Health Syst Pharm 2001, 58(9):784-790.

26. Consolidated Salary Scales in Accordance with Financial Emergency Measures in the Public Interest Act 2013. http://www.hse.ie/eng/staff/ Benefits_Services/pay/July\%202013.pdf.

27. Health Information and Quality Authority: Guidelines for the Budget Impact Analysis of Health Technologies in Ireland. Dublin: Health Information and Quality Authority; 2010.

28. Department of An Taoiseach: How to Conduct a Regulatory Impact Analysis. Dublin: Department of an Taoiseach; 2009. http://www.taoiseach.gov.ie/ eng/Publications/Publications_Archive/Publications_2011/Revised_RIA_ Guidelines_June_2009.pdf.

29. Health Information and Quality Authority: Guidelines for the evaluation of health technologies in Ireland. Dublin: Health Information and Quality Authority; 2010. http://www.hiqa.ie/healthcare/health-technology-assessment/ guidelines.z.

30. Rottenkolber D, Hasford J, Stausberg J: Costs of adverse drug events in German hospitals-a microcosting study. Value Health 2012, 15(6):868-875. 
31. Bates DW, Spell N, Cullen DJ, Burdick E, Laird N, Petersen LA, Small SD, Sweitzer BJ, Leape LL, Adverse Drug Events Prevention Study Group: The costs of adverse drug events in hospitalized patients. JAMA 1997, 277(4):307-311.

32. Heerey A, McGowan B, Ryan M, Barry M: Microcosting versus DRGs in the provision of cost estimates for use in pharmacoeconomic evaluation. Expert Rev Pharmacoecon Outcomes Res 2002, 2(1):29-33.

33. National Casemix Programme: Ready Reckoner of Acute Hospital inpatient and day case activity and costs (summarised by DRG) relating to 2011 costs and activity. Ireland: Health Service Executive; 2013.

34. CA R: Arzneimittelmanagement im Krankenhaus. Eine theoretische und empirische Analyse. Lang: Frankfurt am Main; 2007.

35. Anderson R: Systematic reviews of economic evaluations: utility or futility? Health Econ 2010, 19(3):350-364.

36. Anderson SV, Schumock GT: Evaluation and justification of clinical pharmacy services. Expert Rev Pharmacoecon Outcomes Res 2009, 9(6):539-545.

37. Galvin M, Jago-Byrne MC, Fitzsimons M, Grimes T: Clinical pharmacist's contribution to medication reconciliation on admission to hospital in Ireland. Int J Clin Pharm 2013, 35(1):14-21.

38. Bracey G, Miller G, Franklin BD, Jacklin A, Gaskin G: The contribution of a pharmacy admissions service to patient care. Clin Med 2008, 8(1):53-57.

39. Tam VC, Knowles SR, Cornish PL, Fine N, Marchesano R, Etchells EE: Frequency, type and clinical importance of medication history errors at admission to hospital: a systematic review. CMAJ 2005, 173(5):510-515.

40. International Conference on Harmonisation of Technical Requirements for Registration of Pharmaceuticals for Human Use (ICH): Post-Approval Safety Data Management: Definitions and Standards for Expedited Reporting E2D. Genève: ICH Secretariat; 2003

41. Patanwala $A E$, Hays DP, Sanders $A B$, Erstad BL: Severity and probability of harm of medication errors intercepted by an emergency department pharmacist. Int J Pharm Pract 2011, 19(5):358-362.

42. Paradis AR, Stewart VT, Bayley KB, Brown A, Bennett AJ: Excess cost and length of stay associated with voluntary patient safety event reports in hospitals. Am J Med Qual 2009, 24(1):53-60.

43. Molloy D, Molloy A, O'Loughlin C, Falconer M, Hennessy M: Inappropriate use of proton pump inhibitors. Ir J Med Sci 2010, 179(1):73-75.

44. Cahir C, Fahey T, Tilson L, Teljeur C, Bennett K: Proton pump inhibitors: potential cost reductions by applying prescribing guidelines. BMC Health Serv Res 2012, 12:408.

45. Crowley RK, Fitzpatrick F, Solanki D, FitzGerald S, Humphreys H, Smyth EG: Vancomycin administration: the impact of multidisciplinary interventions. J Clin Pathol 2007, 60(10):1155-1159.

46. Dunn K, O'Reilly A, Silke B, Rogers T, Bergin C: Implementing a pharmacist-led sequential antimicrobial therapy strategy: a controlled before-and-after study. Int I Clin Pharm 2011, 33(2):208-214.

\section{Submit your next manuscript to BioMed Central and take full advantage of:}

- Convenient online submission

- Thorough peer review

- No space constraints or color figure charges

- Immediate publication on acceptance

- Inclusion in PubMed, CAS, Scopus and Google Scholar

- Research which is freely available for redistribution 